\title{
Commissioning and quality control of a dedicated wide bore 3T MRI simulator for radiotherapy planning
}

\author{
Aitang Xing1,4, Lois Holloway1,2,3,4, Sankar Arumugam1,4, Amy Walker 1,3, Robba Rai1, \\ Ewa Juresic ${ }^{1}$, Lynette Cassapi1 ${ }^{1}$, Gary Goozee ${ }^{1,4}$, Gary Liney ${ }^{1,3,4}$ \\ ${ }^{1}$ Liverpool and Macarthur Cancer Therapy Centres \& Ingham Institute, Liverpool Hospital, Sydney, Australia \\ 2Institute of Medical Physics, School of Physics, University of Sydney, Sydney, Australia \\ ${ }^{3}$ Centre for Medical Radiation Physics, University of Wollongong, Wollongong, Australia \\ ${ }^{4}$ South Western Sydney Clinical School, University of New South Wales, Sydney, New South Wales, Australia
}

Received October 29, 2015; Revised February 12, 2016; Accepted February 23, 2016; Published Online March 03, 2016

\section{Original Article}

\begin{abstract}
Purpose: The purpose of this paper is to describe a practical approach to commissioning and quality assurance $(\mathrm{QA})$ of a dedicated wide-bore 3 Tesla (3T) magnetic resonance imaging (MRI) scanner for radiotherapy planning. Methods: A comprehensive commissioning protocol focusing on radiotherapy (RT) specific requirements was developed and performed. RT specific tests included: uniformity characteristics of radio-frequency (RF) coil, couch top attenuation, geometric distortion, laser and couch movement and an end-to-end radiotherapy treatment planning test. General tests for overall system performance and safety measurements were also performed. Results: The use of pre-scan based intensity correction increased the uniformity from $61.7 \%$ to $97 \%$ (body flexible coil), from $50 \%$ to $90 \%$ (large flexible coil) and from 51\% to $98 \%$ (small flexible coil). RT flat top couch decreased signal-to-noise ratio (SNR) by an average of $42 \%$. The mean and maximum geometric distortion was found to be $1.25 \mathrm{~mm}$ and $4.08 \mathrm{~mm}$ for three dimensional (3D) corrected image acquisition, $2.07 \mathrm{~mm}$ and $7.88 \mathrm{~mm}$ for two dimensional (2D) corrected image acquisition over $500 \mathrm{~mm} \times 375 \mathrm{~mm} \times 252 \mathrm{~mm}$ field of view (FOV). The accuracy of the laser and couch movement was less than $\pm 1 \mathrm{~mm}$. The standard deviation of registration parameters for the end-to-end test was less than $0.41 \mathrm{~mm}$. An on-going QA program was developed to monitor the system's performance. Conclusion: A number of RT specific tests have been described for commissioning and subsequent performance monitoring of a dedicated MRI simulator (MRI-Sim). These tests have been important in establishing and maintaining its operation for RT planning.
\end{abstract}

Keywords: MRI in Radiotherapy; Wide-Bore 3T MRI Scanner; Radiotherapy Planning; Quality Control

\section{Introduction}

Computed tomography (CT) has been the primary imaging modality in radiation oncology over several decades due to its excellent spatial resolution, high geometric integrity, short exam time and the use in establishing electron density information required for dose calculation. ${ }^{1}$ However, magnetic resonance imaging (MRI) has a number of advantages over CT for radiotherapy (RT) planning including excellent soft-tissue contrast and the use of non-ionizing radiation. ${ }^{2,3}$
MRI has been used in radiotherapy planning since the $1980 s^{4}$, but images were usually acquired with a diagnostic scanner.5,6 In the last several years, MRI scanners that are designed for radiotherapy simulation purposes (also known as a MRI simulator or MRI-Sim) have become commercially available from several vendors. $^{7-10}$ The dedicated MRI-Sim has several design features, which are unique and considered to be essential for radiotherapy treatment simulation such as a flat couch top, RT specific scanning protocols, an external laser system and flexible coils suitable for 
imaging patients at radiotherapy setup positions. With these technical advances, more oncology centres are expected to install a dedicated scanner in the coming years.

However, the installation, commissioning and quality control of a MRI-Sim within a radiotherapy department can be a challenge. Unlike CT simulator (CT-Sim) ${ }^{11-15}$, there are no well-established commissioning procedures and protocols for a MRI-Sim. Although a set of standard tests are recommended by The American association of Physicists in Medicine (AAPM) ${ }^{16}$ and Institute of Physics and Engineering in Medicine (IPEM) ${ }^{\mathbf{1 7}}$, they were mainly aimed at diagnostic purposes for radiology departments. Staff working in an oncology centre (eg. radiation oncology medical physicists, radiation therapists and oncologists) usually have limited skills and experience with MRI.

As far as the authors know, there is no literature describing the procedure and methodology for acceptance testing and commissioning of a 3 Tesla (3T) MRI simulator in detail. Recently Paulson et al. reported their experiences of using a 3T MRI scanner in radiotherapy, however this work mainly focused on the development of MRI scanning protocols and simulation procedures. $^{7}$ A list of tests was performed by Glide-Hurst et al. to commission a low field (1T) open bore MRI-Sim ${ }^{8}$, but more conventional systems with a wide closed bore at higher field strengths offer the benefits of improved signal-to-noise ratio (SNR) and reduced distortion and was a preferred choice for utilizing MRI in radiotherapy planning. ${ }^{10}$ Although commissioning a 3T MRI scanner for radiotherapy planning may share some tests as used for commissioning an 1T open-bore MRI-Sim, the stronger magnetic field and closed bore structure present different challenges. There are also several other issues, which have not been addressed in published literature such as the MRI safety issue posed by a strong magnetic field and its location in a radiotherapy environment, characterization of radio-frequency (RF) coils, as well as the tests included and frequencies used in an on-going quality assurance (QA) program.

The aim of this paper is twofold: (1) to share our commissioning experience with the radiation oncology community considering the use of MRI in radiotherapy; (2) to report the practical methodologies for addressing these issues and the tests performed during installation, acceptance tests, commissioning and quality control of a wide-bore 3T MRI-Sim installed in an oncology department.

\section{Methods and Materials}

\subsection{Dedicated 3T MRI simulator}

A wide-bore Siemens Skyra 3T MRI scanner (Siemens Healthcare, Erlangen, Germany) equipped with XQ gradients (amplitude: $45 \mathrm{mT} / \mathrm{mm}$ and Slew rate: 200 $\mathrm{T} / \mathrm{mm} / \mathrm{s}$ ), as shown in Figure 1, was installed in July 2013. This magnet is cylindrically optimized with a 70 $\mathrm{cm}$ diameter patient bore to accommodate immobilization devices. The system was configured with a number of radiotherapy simulation features. The localization lasers mounted on an external goalpost were included, which were considered essential for radiotherapy simulation and in particular for MRI-only planning. The flat couch top (Model: MTM3002, Civco Medical Solution, USA) was matched with the couches used on both our linear accelerators (Elekta ${ }^{\circledR} \mathrm{AB}$, Sweden) and TomoTherapy unit (Accuray ${ }^{\circledR}$, Madison, USA). A 32-channel spine coil is integrated into the patient table to improve the image quality for a variety of treatment sites when used in combination with other coils. A range of additional RF coils were selected with the purpose of oncology simulation in mind: a 20 channel head and neck coil, a 16 channel breast coil, two 18 channel body array coils, two large and two small 4 channel flexible coils. Both a long and short cable length version of the body coil was selected, making it possible to connect the coils to the ports at either end of the couch and permit extensive coil coverage in certain setups. A non-standard transmitter/receiver (T/R) head-neck coil was also purchased separately for some of the quality assurance tests.

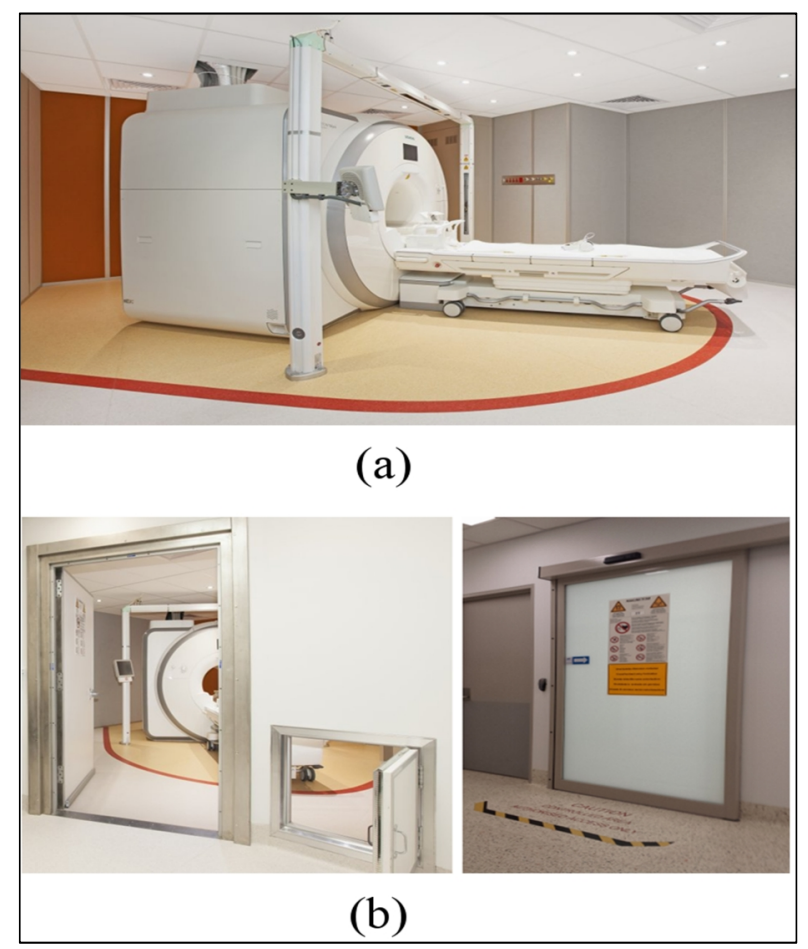

Figure 1: (a) The photo of Siemens Skyra 3T MRI simulator with a flat couch top and stand post supporting simulation lasers. The 30-Gauss line was marked on the floor around the scanner; (b) (Left) Inward-open door between control room and scanner. (Right) The access door to control room from the corridor with the warnings on the door and the ground. 


\subsubsection{Site planning and installation}

The chosen location for the MR-Sim was an old x-ray simulator room next to the CT-Sim. This room was not specifically designed for MRI, and is relatively small. There is an inpatient waiting area and nurse bay with life support equipment on one side of the room and a busy corridor on the other side. To address some of the safety and logistical challenges, the following considerations were made: (a) the magnet was positioned at an angle towards the door to maximize space for equipment and permit the use of the dockable couch in and out of the room. (b) With the magnet in this position, a camera is used at either end of the bore for viewing the patient from the control room. An external window was added inside the room to improve lighting. Extensive passive shielding was used in the walls near the corridors and adjacent public areas to ensure the 5 Gauss limit within the room; (c) An area marking 30
Gauss (proximity limit for non-unrestrained ferromagnetic objects) was highlighted as part of the floor design. (d) A pressure release hatch was included in the RF cage to account for the inward opening of the scan room door in case of a non-vented quench. (e) A card controlled sliding door was installed to restrict entry to the MRI control room from the corridor. Acoustic dampening material was used in the walls to reduce the noise within the department.

\subsubsection{Safety and RF shielding}

Shortly after installation the fringe field was measured using a handheld Gauss meter at various locations around the MRI-Sim (Figure 2). The effectiveness of the $\mathrm{RF}$ cage was verified by measuring the RF attenuation of an RF source placed in the control room and a receiver positioned at various locations inside the scan room.

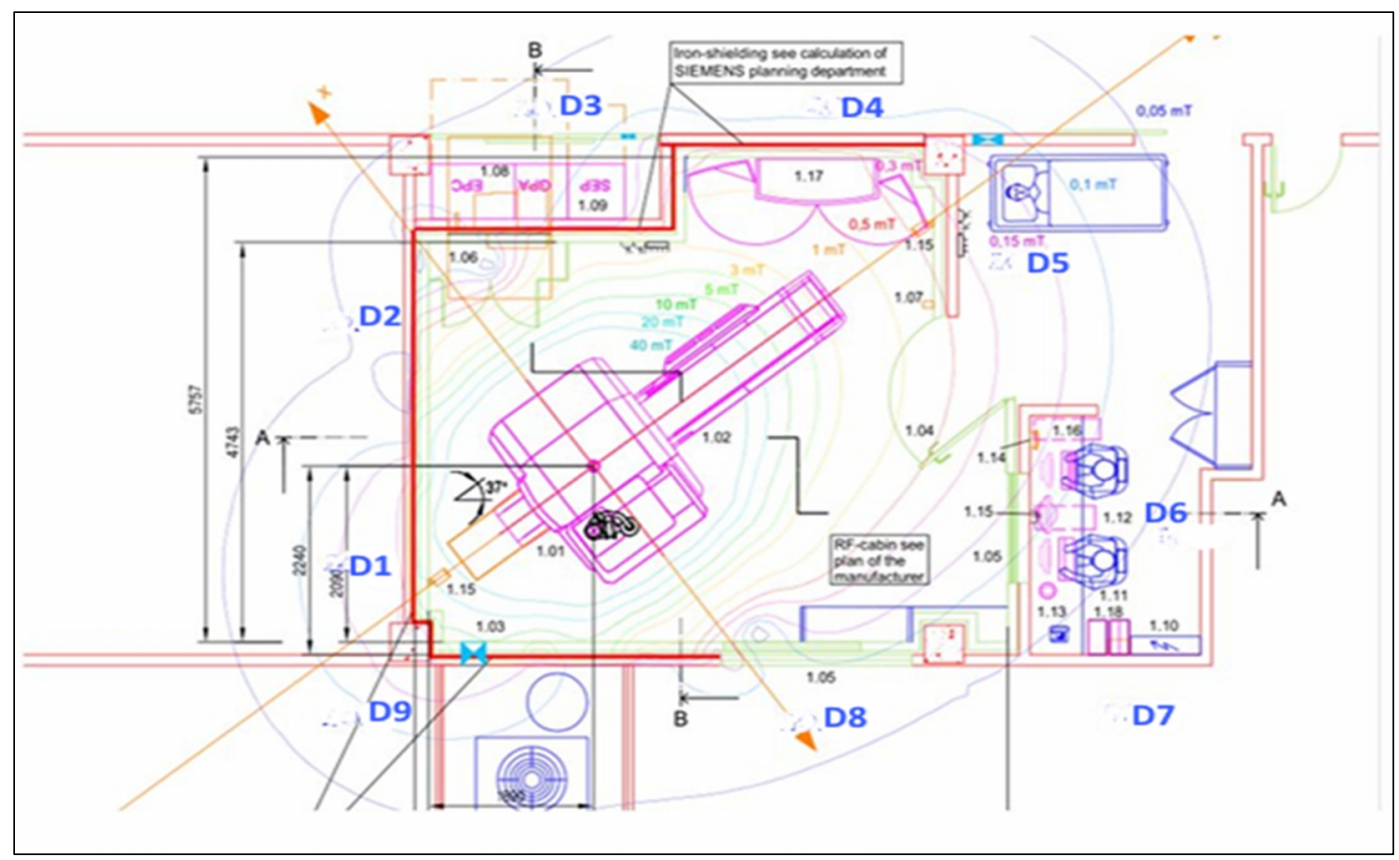

Figure 2: Plane view of the scanner showing its relationship with adjacent rooms and the vendor's predicted field contours. Locations of fringe field measurements are indicated by D1 to D9.

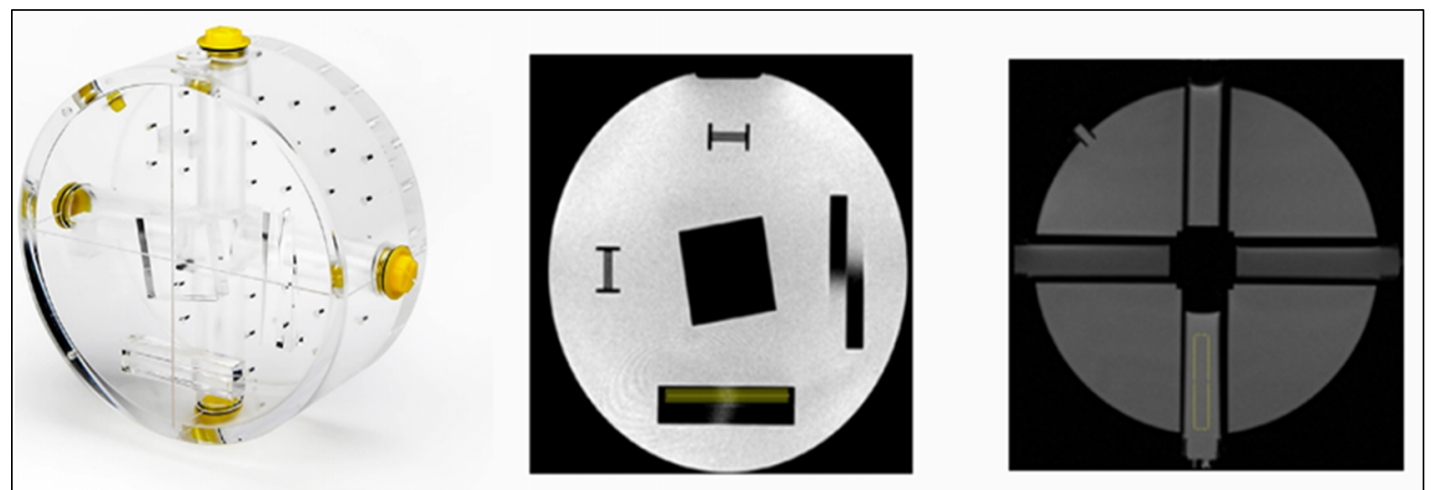

Figure 3: (Left to right) Photos of LTO phantom, the image section used for slice thickness measurement and MTF calculation and the section for contrast factor measurement. 


\subsubsection{System performance}

A series of tests were undertaken following the previous guidelines for quality control of a diagnostic MRI scanner ${ }^{17}$ and are only briefly described here. These measurements were made with the 'all-in-one' MagIQ phantom (Leeds Test Objects (LTO)) using the T/R head coil. This test object is a $10 \mathrm{~cm}$ long cylindrical phantom with a diameter of $23 \mathrm{~cm}$ and filled with mineral oil to reduce non-uniformities due to the increased dielectric effect at high field. It contains a number of features in various sections to enable multiple image metrics to be measured in one acquisition. As shown in Figure 3, the section used for slice profile and resolution contains a pair of oppositely angled plates from which the slice profile can be estimated. The vertical and horizontal bar patterns with a thickness/spacing of $0.5 \mathrm{~mm}$ to provide a quick visual check of resolution in cross-plane and in-plane axes. In addition, an angled block is used to quantify the modulation transfer function (MTF). The user-defined test-tube section was filled with doped (1.5 $\mathrm{T}$ solution) and un-doped water to provide a signal contrast measure. The phantom also contains a flood-fill area for measuring SNR, uniformity and ghosting and a grid pattern to evaluate geometric distortion albeit over a small area. The percentage geometric distortion $(\mathrm{PGD})^{16}$ was calculated using the actual and measured grid positions.

\subsection{RT specific tests}

\subsubsection{RF coil uniformity}

RF coil sensitivity leads to image non-uniformity and is particularly problematic with surface coils and arrays often used to image at the RT position. Two types of corrections were investigated: Pre-scan normalization and B1 filter. Pre-scan normalisation uses an inherently uniform image acquired with the integrated body coil during pre-scan as the basis for correcting non-uniform signal in the subsequent image. This correction can be applied with normal or broad range settings. B1 filter is a post-processing image filter, which can be applied with either a medium or strong setting. A $17 \mathrm{~cm}$ cylindrical phantom filled with NiSO4 solution was imaged using the following coils: body flexible coil, a pair of laterally placed large flexible coils, a similar arrangement of two small flexible coils and the table (spine) coil. For each coil, images were acquired without and with correction using different settings. The uniformity was measured using a whole region of interest (ROI) and a pixel-by-pixel method. ${ }^{5}$ In the whole region method, the percentage uniformity was calculated as

$$
U(\%)=100 \times(1-\sigma / S)
$$

The factional uniformity was calculated as

$$
\begin{aligned}
& U=1-A D D / S, \\
& A D D=\sum_{i=1}^{n}\left(\left|S_{i}-S\right|\right) / n
\end{aligned}
$$

where, $S$ is the mean signal intensity and $S_{i}$ is the intensity of the $i^{\text {th }}$ pixel for a total of $n$ pixels.

To permit a visual assessment, uniformity maps were also generated by comparing $S_{i}$ with $S$ on a pixel-by-pixel basis.

The SNR was calculated as

$$
S N R=\sqrt{2-\pi / 2} S / \sigma
$$

Where, $\sigma$ is the standard deviation.

Signal ghosting, $G(\%)$, was determined as follows:

$$
G(\%)=100 \times\left(B_{P E}-B\right) / S
$$

Where $B_{P E}$ and $B$ are the background signal along the phase and frequency directions respectively. $S$ is the averaged signal of the ROI.

\subsubsection{Flat couch top}

The flat couch top is a $14 \mathrm{~cm}_{\text {IPPS }}^{\text {тм }}$ indexing system, whose MRI Lock-Bar is fixed onto the dockable MRI patient table. The accuracy of the couch movement was verified in the longitudinal direction by comparing the distance shown on the bore display panel to the measured distance using a ruler lying on the couch top. Other mechanical checks included locking of the couch top, docking and home positioning of the patient table. The couch top was designed to fit over the 32 channel $\mathrm{RF}$ coil which was built into the table. To assess its impact on the image quality, the $17 \mathrm{~cm}$ cylinder phantom was positioned over the fourth element of the spine coil (SP4) and imaged with and without flat couch top. SNR and uniformity were calculated as described previously.

\subsubsection{Geometric distortion}

To evaluate the geometric distortion over a large field of view, an in-house made phantom was used. The construction of this phantom was described previously. ${ }^{18}$ Briefly, this is a multi-layered phantom, consisting of polypropylene sheets and a grid pattern of vitamin E capsules. Compared with other commercially available phantoms, this phantom covers a larger volume with several thousand measurement points to permit a detailed measurement of the scanner bore. The phantom was positioned at the isocenter and scanned using two dimensional (2D) image acquisition sequences. During the scanning, the vendor-provided 2D or three dimensional (3D) distortion corrections were applied.

\subsubsection{Simulation lasers}

The external lasers are the LAP lasers mounted on a DORADO nova magnetic resonance (MR) 3T Bridge (LAP Gmbh Laser, Germany). The lasers can be moved in three directions using a terminal inside the MRI scanner room, which is connected to its controller located in the MRI control room through an optical fibre. The LAP lasers were adjusted to be coincident with bore lasers during installation. The LAP lasers are used to set up the 
patient, whereas the MRI bore lasers are used to define the isocenter of the imaging volume. The accuracy of laser movement specified by the manufacturer was \pm 0.5 $\mathrm{mm}$. For radiotherapy purposes, a tolerance of $+/-1 \mathrm{~mm}$ was used. The laser was moved over various distances within a $50 \mathrm{~cm}$ range. The actual moving distance was verified using a plastic ruler taped onto either the couch or bridge post.

The distance between the planes defined by the bore and LAP laser and the magnetic isocenter inside the bore were checked using a laser phantom as shown in Figure 4. This removable phantom sits on a base platform with three adjustable legs and two embedded spirit levels for levelling. On the phantom surface, there are alignment markings. Inside the phantom, two small cross tubes filled with high contrast solution are located at the centre of the phantom. The phantom was first aligned with the LAP and bore lasers, then scanned using integrated body coil and a spin echo (SE) sequence. As shown in Figure 4, the appearance of a cross-hair at the centre slice indicated the parallel between isocenter plane and the plane defined by LAP lasers.

\subsubsection{MRI-Sim workflow}

MRI-Sim was integrated into our clinical workflow in the following way: patient is scanned on a CT-Sim at treatment position, and then scanned at same position on the MRI simulator; MRI and CT images are transferred into treatment planning system (TPS). MRI and CT images are fused together; target is contoured on MRI and copied to CT dataset for planning. To test the consistency of this workflow, the laser phantom was scanned on CT-Sim and MRI-Sim. MRI and CT images were transferred to the Pinnacle TPS19 and automatically registered using the mutual information algorithm. The registration parameters were recorded and used as end-point indicators for the whole workflow. This procedure was repeated over a period of 8 days to simulate a fractionated treatment scheme.

\subsubsection{QA program development}

Based on the tests performed during the commissioning period, a QA program was developed. According to the frequency of the tests, the program was divided into daily, monthly, quarterly and annual QA. The daily QA program consisted of two separate tests: laser alignment and image quality. The second test utilized the vendor provided semi-automated customer QA of the head coil using the cylindrical test object described earlier. The result of this test is displayed simply as a pass or fail. The quantitative values of SNR can also be logged. The external and bore laser alignment was checked using the laser phantom. Each of the clinically used coils are tested every month by imaging uniform phantoms. The RT workflow was checked quarterly. On an annual basis, geometric distortion and system performance are measured.

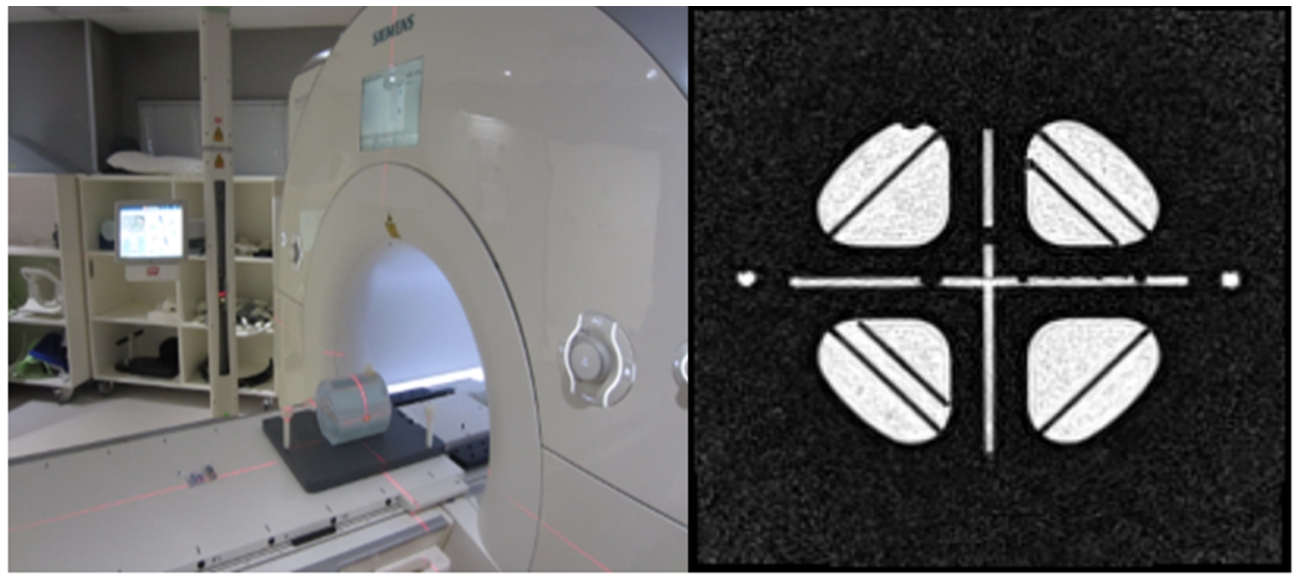

Figure 4: (Left) The laser phantom was set up on the couch top using the external simulation laser controlled by the LAP laser terminal inside the scanner room. (Right) The centered transverse slice of laser phantom images showing the cross line. The MRI marker on the phantom surface was visually seen on the images and used for checking image transfer and orientation.

Table 1: Measured fringe field strength at various locations outside the scanner room.

\begin{tabular}{llllllllll}
\hline \hline Locations & D1 & D2 & D3 & D4 & D5 & D6 & D7 & D8 & D9 \\
\hline B (Gauss) & 2 & 0.3 & 3 & 2.4 & 1.7 & 1.5 & 1.8 & 2.8 & 1.8 \\
\hline \hline
\end{tabular}

Table 2: Image quality indices measured using LTO phantom and T/R head coil.

\begin{tabular}{llllllll}
\hline \hline Quality indices & SNR & U(\%) & Contrast & Ghosting & Resolution(F50) & Slice thickness(mm) & PGD(\%) \\
\hline Measured values & 65 & 93 & 0.28 & 0.04 & 0.9 & 2.72 & 0.6 \\
Criteria & N/A & $>90$ & N/A & $<1$ & N/A & $2.7-3.3$ & $<2$ \\
\hline \hline
\end{tabular}




\section{Results}

\subsection{Safety and RF shielding}

The 5-Gauss line was verified as being confined by the scan room perimeter and a small external fenced area outside. The actual measured magnetic field established with a survey meter at various locations outside the scan room was lower than the value from the expected fringe field as shown in Table 1. The RF attenuation was quantified in terms of $\mathrm{dB}$ where a high value indicates greater attenuation and therefore less noise transmitted through the RF cage. The minimal attention recommended by the manufacturer is $90 \mathrm{~dB}$. The measured attenuation was between $96 \mathrm{~dB}$ and $110 \mathrm{~dB}$ over the desired frequency range (64 to $128 \mathrm{MHz}$ ). Acoustic noise reduction, although not quantified, was noted as being effective with little discernible noise in the control room and adjacent corridor while the scanner is running.

\subsection{Overall system performance}

Table 2 showed the general image test results calculated from different sections of the LTO phantom. Modulation transfer function was calculated and the $50 \%$ value (F50) was used as a reference value for image resolution. The measured slice thickness corresponds to the nominal thickness of $3 \mathrm{~mm}$. The acceptance criteria, where applicable, for commissioning a diagnostic scanner is also shown here for reference. ${ }^{16}$

\subsection{RF coil uniformity characteristics}

The effect of the pre-scan normalize filter and B1 filter on image quality was presented in Table 3 and plotted in Figure 5. The uniformity increased from $61.7 \%$ to $97 \%$ for Body flex, from $50 \%$ to $90 \%$ for large flex and from $51 \%$ to $98 \%$ for small flex by applying the pre-scan normalize filter with the broad range setting. The filter also enhances the SNR for all three coils. For example, SNR for the body flex coil increases from 269 to 290. The pre-scan normalize filter strength has no effect on the body flex coil's uniformity, but improved SNR by 1.3 and 1.2 times for the large and small flex coils, respectively. The B1 filter was found to have a negligible effect on image uniformity, but it affected SNR. When the B1 filter was applied with medium strength setting, SNR decreased from 268.3 to 250 for the flexible body coil and increased from 167 to 209 for large flexible coil. The ghosting effect became worse for the large and small flexible coils when either the B1 filter or pre-scan normalize filter were applied.

\subsection{Flat couch top}

The effect of the couch top on the image quality was shown in Table 4 and Figure 5 (b). The couch top reduced the MRI signal, irrespective of the filter used. The SNR was decreased by $41.2 \%$ and $42.8 \%$ for pre-scan normalize filter with normal strength and broad range settings, respectively when the couch top was placed on the bed. However, the image uniformity was improved slightly with the couch top in situ. The uniformity was increased by $15.8 \%$ when the normal pre-scan normalize filter was used and $10.9 \%$ when the pre-scan normalize filter with broad range strength was applied. The ghosting effect may decrease or increase depending on the filter settings.

Table 3: The impact of various filters on image quality with different strengths.

\begin{tabular}{llllll}
\hline \hline $\begin{array}{l}\text { Applied } \\
\text { filters }\end{array}$ & Strength & Metric & $\begin{array}{l}\text { Body } \\
\text { flex }\end{array}$ & $\begin{array}{l}\text { Large } \\
\text { flex }\end{array}$ & $\begin{array}{l}\text { Small } \\
\text { flex }\end{array}$ \\
\hline No filter & & SNR & 268.3 & 167 & 189 \\
& & G (\%) & 3.3 & 1.18 & 1.03 \\
Pre-scan & Normal & SNR & 312 & 251 & 340 \\
normalize & & G (\%) & 0.21 & 2.15 & 3.68 \\
filter & & U (\%) & 97 & 68 & 85 \\
& Broad & SNR & 290 & 247 & 227 \\
& Range & G (\%) & 3.38 & 4.16 & 4.37 \\
& & U (\%) & 97 & 90 & 98 \\
B1 filter & Medium & SNR & 250 & 209 & 161 \\
& & G (\%) & 1.56 & 5.78 & 3.6 \\
& & U (\%) & 85 & 87 & 82 \\
& Strong & SNR & 269 & 214 & 170 \\
& & G (\%) & 1.97 & 5.6 & 5.17 \\
& & U (\%) & 84 & 87 & 83 \\
\hline \hline
\end{tabular}

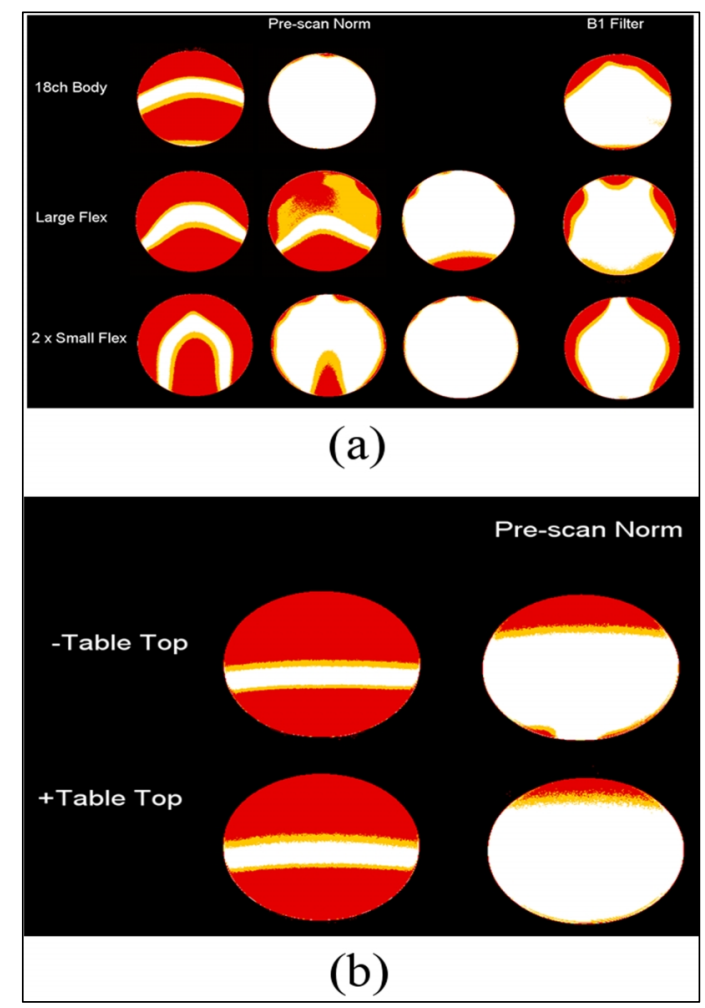

Figure 5: (a) Uniformity map for each of the RF coils with different filter applied: no filter, pre-scan filter with normal setting, pre-scan filter with broad range settings, and the B1 filter with medium setting. (b) Maps of uniformity for the fourth spinal coil (SP4) without filter and with the pre-scan normalize filter using the broad range setting and with and 
without table top. Values are displayed as three colour levels with white, yellow and red corresponding to $\pm 20 \%$, $\pm 30 \%$ and $>30 \%$ respectively.

Table 4: The measured image quality metrics for the integrated table coil with and without flat couch top.

\begin{tabular}{lcccc}
\hline \hline \multirow{2}{*}{ Metric } & \multicolumn{2}{c}{ Couch top removed } & & \multicolumn{2}{c}{ Couch top in situ } \\
\cline { 2 - 5 } & Normal & Broad range & Normal & Broad range \\
\hline SNR & 223 & 168 & 131 & 96 \\
$\mathrm{G}(\%)$ & 1.93 & 1.02 & 1.37 & 1.24 \\
$\mathrm{U}(\%)$ & 32 & 82 & 38 & 91 \\
$\mathrm{U}$ & 0.43 & 0.85 & 0.5 & 0.91 \\
\hline \hline
\end{tabular}

\subsection{Geometric distortion}

Table 5 presents the geometric distortion after the system correction algorithm was applied. For 2D corrected images, distortion of up to $7.9 \mathrm{~mm}$ (mean $=2.1$ $\mathrm{mm}$ ) was observed. This was reduced to $4.1 \mathrm{~mm}$ (mean $=1.25 \mathrm{~mm}$ ) over a radial distance of $150 \mathrm{~mm}$ from isocenter when 3D correction was used. Detailed 3D distortion maps are shown in Figure 6.

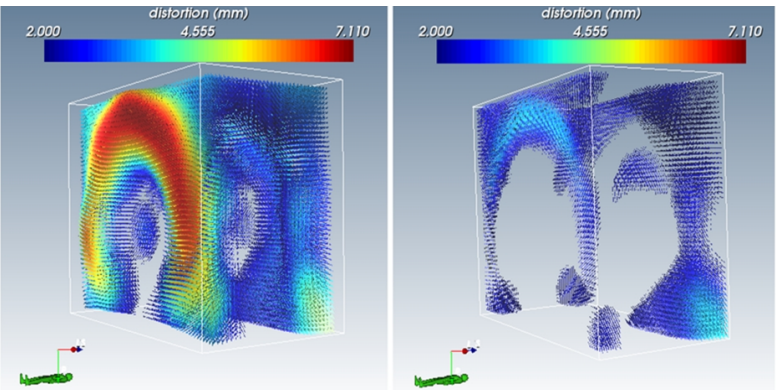

Figure 6: 3D map plot of the geometric distortion after the application of 2D (left) and 3D (right) correction algorithm provided by the vendor.

\subsection{End-to-end test}

The end-to-end test results are shown in Table 6. The translation and rotation coordinates indicate the reproducibility and consistency of RT workflow: positioning and setup of phantom, the daily variation of scanner performance, and the consistency of image registration in Pinnacle. The $11 \mathrm{~cm}$ shift along the y-axis is the distance between LAP and bore lasers. The average translation and rotational errors are less than $1.2 \mathrm{~mm}$ and 0.24 degrees, respectively.

Table 5: Statistics of geometric distortion quantified using the in-house made phantom with system correction turned on.

\begin{tabular}{cccccc}
\hline \hline $\begin{array}{c}\text { Acquisition } \\
\text { mode }\end{array}$ & $\begin{array}{c}\text { Max distance } \\
(\mathrm{mm})\end{array}$ & $\begin{array}{c}\text { Max distortion } \\
(\mathrm{mm})\end{array}$ & $\begin{array}{c}\text { Mean distortion } \\
(\mathrm{mm})\end{array}$ & $\begin{array}{c}\text { Min distortion } \\
(\mathrm{mm})\end{array}$ \\
\hline 2D correction & 98 & 7.88 & 2.07 & 0.04 \\
3D & correction & 152 & 4.08 & 1.25 & 0.05 \\
\hline \hline
\end{tabular}

a: The maximum distance from isocenter where the distortion is less than $2 \mathrm{~mm}$.

Table 6: End-to-end test results for an MRI integrated RT workflow for 8 simulated fractions using the laser phantom.

\begin{tabular}{ccccccc}
\hline \hline & \multicolumn{3}{c}{ Translation(cm) } & \multicolumn{3}{c}{ Rotation(degree) } \\
\cline { 2 - 7 } Fraction number & $\mathrm{X}$ & $\mathrm{Y}$ & $\mathrm{Z}$ & $\mathrm{X}$ & $\mathrm{Y}$ & $\mathrm{Z}$ \\
\hline 1 & 0.37 & -10.92 & -0.32 & 0.24 & 0.14 & 0.04 \\
2 & 0.24 & -11.13 & -0.32 & 0.00 & 0.00 & 0.00 \\
3 & 0.27 & -11.14 & -0.07 & 0.00 & -0.16 & -0.06 \\
4 & -0.49 & -11.18 & -0.69 & 0.00 & 0.82 & -0.06 \\
5 & 0.28 & -11.12 & -0.08 & 0.00 & -0.16 & 0.00 \\
6 & 0.07 & -11.60 & 0.53 & 0.02 & 1.03 & 0.00 \\
7 & 0.28 & -10.91 & -0.33 & 0.00 & -0.06 & 0.00 \\
8 & -0.29 & -10.93 & 0.41 & 0.06 & 0.20 & 0.05 \\
Average & 0.09 & -11.12 & -0.11 & 0.04 & 0.23 & 0.00 \\
Standard deviation & 0.31 & 0.23 & 0.41 & 0.08 & 0.45 & 0.04 \\
\hline \hline
\end{tabular}

Table 7: Quality assurance protocols developed for monitoring system's performance.

\begin{tabular}{lllll}
\hline \hline Frequency & Checked items & Devices & Quantities & Tolerance \\
\hline Daily QA & Lap laser alignment & Laser phantom & Alignment & $\pm 1 \mathrm{~mm}$ \\
& System consistency & Cylindrical phantom & SNR & $\pm 3 \%$ \\
Monthly QA & Laser movement & Rulers & Distance & $\pm 1 \mathrm{~mm}$ \\
& Couch movement & Rulers & Distance & $\pm 1 \mathrm{~mm}$ \\
Quarterly QA & Coil stability & Vendor's phantoms & SNR & $\pm 3 \%$ \\
Annual QA & System performance & LTO phantom & Rotation translation & $\pm 2 \%$ \\
& & & SNR & \\
& & & Contrast & \\
& Geometric distortion & & Ghosting & $\pm 3 \%$ \\
& & In-house phantom & Slice thickness & \\
\hline \hline
\end{tabular}




\subsection{QA program}

Table 7 shows the developed on-going QA protocol along with frequency and tolerance values. For each test, the measured quantities were compared to the baseline values established during commissioning. The coils which are heavily used may be degraded due to physical or electronic damage. Recently the connector used for flexible coils was damaged and this was detected by our monthly QA. A coil consistency check on a monthly basis was considered adequate to maintain optimal performance established during commissioning.

\section{Discussion}

MRI safety can never be overstated in a radiotherapy environment, where radiation safety is well understood, but the imaging modality may not be appreciated by staff. We performed staff training and implemented local work-flow protocols to address this. These safety issues not only affect the cancer patients, the public and staff but also extend to the various radiotherapy equipment. The linear accelerator, CT simulator and dosimetry equipment need to be in regions of less than 1 Gauss. ${ }^{20}$ Independent verification of the fringe field confirmed that the mangetific field was confined within the scan room as recommended by AAPM. ${ }^{16}$

The tests performed during commissioning focused on the use of MRI in radiotherapy. The tests commonly used for a diagnostic scanner were performed as well using the LTO phantom. However, the purpose of all-in-one phantom based tests was to provide a baseline for ongoing monitoring of the system's performance.

According to the international recommendation for acceptance testing and commissioning of a diagnostic scanner ${ }^{16}$, the test results were within recommended ranges. It was noted however that the ghosting level determined for the surface coils, although low, was up to $5 \%$.

Flexible coils are extremely useful in a radiotherapy setting and can be used alone or combined with other coils for most radiotherapy treatment sites. Flexible coils may be placed over radiotherapy immobilization devices and on any part of the body. However, the intensity of images acquired with flexible coils is not uniform, particularly in comparison to images acquired with head and neck coils. Image non-uniformity does not generally have an impact on the images used for diagnostic purposes. However, it is a problem for the use of MRI in radiotherapy, as accurate intensity-based image MRI-CT fusion and segmentation requires uniform MRI images. ${ }^{7}$ The vendor usually supplies filters to correct the coil sensitivity profile. During commissioning, imaging uniformity with and without filters should be investigated for flexible coils and arrangements of these coils. Our investigation showed that the pre-scan normalize filters improved image uniformity and SNR.
External lasers and a flat couch top are not required for a diagnostic scanner, but they are the critical components for a therapeutic scanner to ensure setting up the patient at same treatment position in MRI-Sim, CT-Sim and treatment unit.2,3 The downside of a flat couch is that it may attenuate the RF signal, as demonstrated in this investigation and the image quality may also be degraded. It is recommended that the effect of the couch top on image quality be investigated for all flat couches used in this manner. For our couch, the reduction of SNR did not adversely affect the routine simulation images, however, this needs to be considered carefully when using other signal challenging sequences such as diffusion weighted imaging (DWI). ${ }^{21}$

Geometric distortion can be classified into patient-related and system-level distortion. ${ }^{18,22,23}$ Patient-related distortion is due to the perturbation of the static field by chemical shift and susceptibility difference of two different tissues, with patient specific amplitude. System level geometric distortion is caused by gradient non-linearity and B0 inhomogeneity. To reduce the machine-related geometric distortion, the vendors provided correction algorithms, which were built into the system. Although the distortion caused by B0 inhomogeneity and gradient non-linearity can be measured separately using a gradient reverse technique 24,25 , for the purpose of radiotherapy the overall amplitude of geometric distortion over a large volume directly affects the accuracy of tumour delineation and should be measured at commissioning stage using a phantom with a grid arrangement of points. The commercial phantoms, such as American college of Radiology (ACR) phantom and LTO, have been commonly used to measure the geometric distortion for a diagnostic scanner ${ }^{26,27}$, however, their size is smaller in one or two directions compared to therapeutic scans and thus an in-house phantom was developed and used for this purpose. For the purpose of commissioning a MRI-Sim, the phantom volume should approximately cover the useable volume within the bore so that the limits of acceptable distortion can be established. Our measurements showed that the 3D corrections were superior to 2D corrections. The 3D correction algorithm was applied when the patient was being scanned in our clinic.

Although the feasibility of a MRI-only RT workflow ${ }^{28-30}$ has been demonstrated, the routine clinical practice of incorporating MRI into radiotherapy is a two-step process: co-registration of MRI images to CT images, target contoured on MRI and copied to CT images. This workflow was verified as a part of commissioning tests using the laser phantom. Many other phantoms could also be used as long as they were both MRI and CT compatible. If deformable registration is used to fuse MRI and CT images, an anthropomorphic phantom may also be useful for this test. ${ }^{31}$ The purpose of this check 
was to verify: (a) The distance between the tranverse planes defined by isocenter and simulation lasers; (b) The reproducibility of patient or phantom setup; (c) The stability of MRI imaging system; (d) The image transfer from MRI console to TPS; (e) The accuracy and consistency of auto-registration in treatment planning system.

An on-going QA program was required to maintain the simulator performance established at the commissioning stage. Like other imaging equipment used in radiotherapy, the stability and consistency of the MRI system needed to be monitored by a series of tests performed at different frequencies. Currently there are no international recommendations on what kind of tests should be performed and the tolerance to be used. The selection of tests and tolerance was mainly based on the observation of system performance over last two years. Well-established protocols for other radiotherapy equipment such CT-Sim was also referenced. Compared with QA programs for a diagnostic MRI scanner ${ }^{16,17,32,33}$, this program was designed to meet the requirements for radiotherapeutic purposes.

Development of a comprehensive commissioning protocol involving a multi-disciplinary team prior to installation is strongly recommended for smoothly integrating MRI-Sim into an existing clinical workflow. It is especially required for oncology centres whose staff have limited MRI experience. A standardized commissioning protocol for a MRI-Sim needs to be established as the number of oncology centres using dedicated MRI-Sim for radiotherapy planning is rapidly increasing.

\section{Conclusion}

Comprehensive tests which were identified as directly related to the use of MRI in radiotherapy were performed. An MRI-Sim was successfully integrated into our clinic and has been monitored by an on-going QA program. MRI-based radiotherapy planning has been performed for 1300 patients for various treatment sites over the first 29 months of operation of our MRI-Sim.

\section{Conflict of interest}

The authors declare that they have no conflicts of interest. The authors alone are responsible for the content and writing of the paper.

\section{References}

1. Aird E, Conway J. CT simulation for radiotherapy treatment planning. Br J Radiol. 2002;75:937-49.

2. Devic S. MRI simulation for radiotherapy treatment planning. Med Phys. 2012; 39:6701-11.

3. Metcalfe P, Liney GP, Holloway L, et al. The potential for an enhanced role for MRI in radiation-therapy treatment planning. Technol Cancer Res Treat. 2013;12:429-46.

4. Fraass B, McShan DL, Diaz RF, et al. Integration of magnetic resonance imaging into radiation therapy treatment planning: I. Technical considerations. Int J Radiat Oncol Biol Phys. 1987; 13:1897-908.

5. Liney GP, Owen SC, Beaumont AK, et al. Commissioning of a new wide-bore MRI scanner for radiotherapy planning of head and neck cancer. Br J Radiol. 2013;86:20130150.

6. Wang C, Chao M, Lee L, Xing L. MRI-based treatment planning with electron density information mapped from CT images: A preliminary study. Technol Cancer Res Treat. 2008;7:341-8.

7. Paulson ES, Erickson B, Schultz C, Li XA. Comprehensive MRI simulation methodology using a dedicated MRI scanner in radiation oncology for external beam radiation treatment planning. Med Phys. 2015;42:28-39.

8. Glide-Hurst CK, Wen N, Hearshen D, et al. Initial clinical experience with a radiation oncology dedicated open 1.0 T MR-simulation. J Appl Clin Med Phys. 2015;16:5201.

9. Mah D, Steckner M, Palacio E, et al. Characteristics and quality assurance of a dedicated open 0.23 T MRI for radiation therapy simulation. Med Phys. 2002;29:2541-7.

10. Liney GP, Moerland MA. Magnetic resonance imaging acquisition techniques for radiotherapy planning. Semin Radiat Oncol. 2014;24:160-8.

11. A Practical Guide to CT Simulation. Editors: Coia LR, Schultheiss TE, Hanks GE. Advanced Medical Publishing, 1995.

12. Ebert MA, Kenny J, Greer PB. Experience converting an RT department to full CT simulation: Technical issues identified during commissioning of a wide-bore scanner. J Med Imaging Radiat Oncol. 2009;53:325-30.

13. McGee K, Das IJ. Commissioning, acceptance testing and quality assurance of a CT simulator. In Coia LR, Schultheiss TE, Hanks GE. A Practical Guide to CT Simulation. 1995:5-23.

14. Zhang J, Sehgal V, Roa DE, et al. SU-GG-J-34: Comprehensive clinical commissioning and quality assurance procedures of a big bore CT simulator in a Radiation Oncology Department. Med Phys. 2010;37:3152-52.

15. Mutic S, Palta JR, Butker EK, et al. Quality assurance for computed-tomography simulators and the computedtomography-simulation process: report of the AAPM Radiation Therapy Committee Task Group No. 66. Med Phys. 2003;30:2762-92.

16. Och JG, Clarke GD, Sobol WT, et al. Acceptance testing of magnetic resonance imaging systems: 
report of AAPM Nuclear Magnetic Resonance Task Group No. 6. Med Phys. 1992;19:217-29.

17. Lerski R, De Wilde J, Boyce D, Ridgeway J. Quality control in magnetic resonance imaging. IPEM Report 80. Institute of Physics and Engineering in Medicine, UK, 1999. ISBN 0-904181 901. European Journal of Radiology, 2000; 36:59- 60.

18. Walker A, Liney G, Holloway L, et al. Continuous table acquisition MRI for radiotherapy treatment planning: Distortion assessment with a new extended 3D volumetric phantom. Med Phys. 2015;42:1982-91.

19. Bedford J, Childs PJ, Nordmark Hansen V, et al. Commissioning and quality assurance of the Pinnacle3 radiotherapy treatment planning system for external beam photons. Br J Radiol. 2003;76:163-76.

20. Dempsey MF, Condon B, Hadley DM. MRI safety review. Semin Ultrasound CT MR. 2002; 23:392-401.

21. Liney GP, Holloway L, Al Harthi TM, et al. Quantitative evaluation of diffusion-weighted imaging techniques for the purposes of radiotherapy planning in the prostate. $\mathrm{Br} \mathrm{J}$ Radiol. 2015;88:20150034.

22. Walker A, Liney G, Metcalfe P, Holloway L. MRI distortion: considerations for MRI based radiotherapy treatment planning. Australas Phys Eng Sci Med. 2014;37:103-13.

23. Vedam S, Docef A, Fix M, et al. Dosimetric impact of geometric errors due to respiratory motion prediction on dynamic multileaf collimator-based four-dimensional radiation delivery. Med Phys. 2005;32:1607-20.

24. Baldwin LN, Wachowicz K, Fallone BG. A two-step scheme for distortion rectification of magnetic resonance images. Medi phys. 2009;
36:3917-26.

25. Stanescu T, Jans HS, Wachowicz K, Fallone BG. Investigation of a $3 \mathrm{D}$ system distortion correction method for MR images. J Appl Clin Med Phys. 2010;11:2961.

26. Chen CC, Wan YL, Wai YY, Liu HL. Quality assurance of clinical MRI scanners using ACR MRI phantom: preliminary results. J Digit Imaging. 2004;17:279-84.

27. Ihalainen TM, Lonnroth NT, Peltonen JI, et al. MRI quality assurance using the ACR phantom in a multi-unit imaging center. Acta Oncol. 2011;50:966-72.

28. Keyriläinen J, Kapanen M, Seppälä T, et al. MRI-only based RTP workflow of prostate cancer patients. Int J Radiat Oncol Biol Phys. 2014;90:S929-30.

29. Brock KK, Dawson LA. Point: Principles of magnetic resonance imaging integration in a computed tomography-based radiotherapy workflow. Semin Radiat Oncol. 2014;24:169-74.

30. Andreasen D, Van Leemput K, Hansen RH, et al. Patch-based generation of a pseudo CT from conventional MRI sequences for MRI-only radiotherapy of the brain. Med phys. 2015; 42:1596-605.

31. Sun J, Dowling J, Pichler P, et al. MRI simulation: end-to-end testing for prostate radiation therapy using geometric pelvic MRI phantoms. Phys Med Biol. 2015;60:3097.

32. Friedman L, Glover GH. Report on a multicenter fMRI quality assurance protocol. J Magn Reson Imaging. 2006;23:827-39.

33. Firbank MJ, Harrison RM, Williams ED, Coulthard A. Quality assurance for MRI: practical experience. Br J Radiol. 2000; 73:376-83. 